\title{
Additive Effect of Brinzolamide on Diurnal Changes in Intraocular Pres- sure in Latanoprost-treated Eyes
}

\author{
Keitetsu Abe ${ }^{1}$ and Kenji Kashiwagi ${ }^{*}, 2$ \\ ${ }^{I}$ Department of Ophthalmology, Yamanashi Prefectural Central Hospital, Kofu, Yamanashi, Japan \\ ${ }^{2}$ Department of Community and Family Medicine, and Department of Ophthalmology Interdisciplinary School of Medi- \\ cine and Engineering, University of Yamanashi, Chuo, Yamanashi, Japan
}

\begin{abstract}
To investigate the effect of brinzolamide on diurnal fluctuations in the intraocular pressure (IOP) in patients on latanoprost ophthalmic solution prospectively was aimed. Eleven patients with primary open angle glaucoma were enrolled in this study. The subjects were admitted to the hospital and had their IOPs measured over 24 hours (10 AM, noon, 2 PM, 4 PM, 6 PM, 8 PM, 10 PM, midnight, 3 AM, 6 AM, and 8AM). After topical administration of brinzolamide twice daily for 4-8 weeks for one eye, they were readmitted and again had their IOPs measured in the same manner as during the previous admission. The additional administration of brinzolamide significantly reduced IOP by $9.7 \pm 4.5 \%$ during 24 hour, $9.5 \pm 4.7 \%$ during daytime, and $10.3 \pm 5.6 \%$ during nighttime, respectively. Brinzolamide exerts additive effects over 24 hours in reducing IOP in patients with glaucoma who are receiving latanoprost ophthalmic solution.
\end{abstract}

Keywords: Brinzolamide, additive effect, diurnal IOP changes, latanoprost, intraocular pressure.

\section{INTRODUCTION}

Intraocular pressure (IOP)-lowering therapy is the most effective and established approach for treatment of glaucoma. There are several options for IOP-lowering therapy: medication, laser therapy and surgical treatment, among which medication is often the first choice for its consistent efficacy and safety. In recent years, it has been thought that maintaining IOP within the normal range is not enough to achieve sufficiently low IOP and it is now required to reach individualized target IOP [1]. Thus, there is growing need for more potent drugs than conventional IOP-lowering therapies.

IOP is known to fluctuate during the day because of diurnal rhythms and it is important to maintain a target IOP throughout a day in order to prevent progression of glaucoma [2,3]. Latanoprost is widely used all over the world as a first-line medication for glaucoma as it has an excellent IOP-lowering effect over 24 hours and is associated with only mild adverse reactions [4]. Latanoprost alone, however, sometimes to fail to achieve sufficiently low IOP, leading many patients to use two or more ophthalmic anti-glaucoma solutions [1]. Particularly in Japan, since there are many patients with normal tension glaucoma (NTG) [5], topical administration of latanoprost alone often fails to produce sufficient IOP-lowering effects.

Brinzolamide is a carbonic anhydrase inhibitor that causes fewer local or systemic adverse reactions [6]. It exerts an excellent IOP-lowering effect by suppressing formation of aqueous humor [7]. It is also known to significantly lower IOP over 24 hours and considered as an adequate ophthalmic solution for control of IOP over 24 hours [6, 8]. In Japan,

*Address correspondence to this author at the Department of Ophthalmology, University of Yamanashi Faculty of Medicine, 1110 Shimokato, Chuo, Yamanashi 409-3898, Japan; Tel: 81-552-73-9657; Fax: 81-552-73-6757; E-mail: kenjik@yamanashi.ac.jp however, brinzolamide is not approved as a first-line drug for glaucoma and in clinical settings, it is often used concomitantly with other ophthalmic solution such as latanoprost. Nevertheless, there have been few studies that examined the IOP-lowering effect of brinzolamide, especially during nighttime [9], when it is concomitantly administered with other drugs. Therefore, we investigated the IOPlowering effect of brinzolamide over 24 hours in patients with glaucoma who had been receiving latanoprost ophthalmic solution and who additionally received brinzolamide in one of their eyes, and evaluated the efficacy of the additional administration of brinzolamide.

\section{SUBJECTS AND METHODS}

This study was conducted in accordance with the Declaration of Helsinki, having appropriate institutional review board approval and the patients' written informed consent.

\section{Subjects}

We studied seven patients with primary open angle glaucoma (POAG) and four patients with NTG who had been receiving latanoprost ophthalmic solution alone in both eyes for at least 4 weeks. Their mean age was 58.1 years ranging from 42 to 73 years, and there were 6 men and 5 women. Patients were excluded from the study if they had uveitis, corneal diseases that might interfere with IOP measurements, a history of intraocular surgery, including laser therapy, or an MD of $-15 \mathrm{~dB}$ or worse on Humphrey visual field test 302. Patients on steroids or oral carbonic anhydrase inhibitor, or those who were deemed inappropriate by the investigator were also excluded from the study. All eligible patients were enrolled in the study from October 2004 to September 2006.

\section{Study Protocol}

All patients received latanoprost ophthalmic solution in both eyes once daily at 9 PM for at least 4 weeks. During 
this period, we confirmed that patient compliance was good and no adverse reactions occurred in association with latanoprost. While on latanoprost, the patients were admitted to the Department of Ophthalmology of Yamanashi Prefectural Central Hospital and had their IOPs measured over 24 hours (10 AM, noon, 2 PM, 4 PM, 6 PM, 8 PM, 10 PM, midnight, $3 \mathrm{AM}, 6 \mathrm{AM}$, and 8AM). Measurements of IOP were taken in the sitting position by ophthalmologists using a Goldmann applanation tonometer. After testing for diurnal fluctuations in IOP, one eye with the higher IOP at $10 \mathrm{AM}$, or the right eye if the IOPs at $10 \mathrm{AM}$ were the same in both eyes, was selected for brinzolamide administration twice daily (7 AM and $7 \mathrm{PM}$ ). After confirming that the additional administration of brinzolamide caused no adverse reactions, the patients were readmitted to the Department of Ophthalmology of Yamanashi Prefectural Central Hospital at week 4-8 of brinzolamide treatment and again had their IOPs measured in the same manner as during the previous admission. During IOP measurements, the ophthalmologists were blinded as to which eye was treated with brinzolamide.

\section{Analyzed Parameters}

The following data were analyzed in this study: mean IOP, maximum IOP, minimum IOP, and a range of fluctuations in IOP in brinzolamide-treated eye and brinzolamideuntreated eye, comparison of IOP values and percent reduction in IOPs at each measurement point before and after brinzolamide administration, comparison of IOP values and percent reduction in IOPs between brinzolamide-treated eye and brinzolamide-untreated eye, and comparison of percent reduction in IOPs between daytime (8 AM, $10 \mathrm{AM}$, noon, 2 $\mathrm{PM}, 4 \mathrm{PM}, 6 \mathrm{PM}$ ) and nighttime (8 PM, $10 \mathrm{PM}$, midnight, 3 AM).

\section{Statistical Tests}

Mann-Whitney U test or paired-t test were employed for statistical analysis and a statistically significant difference was defined as less than 5\%. All data expressed as mean \pm SD.

\section{RESULTS}

\section{Changes in Mean Daily IOP}

In brinzolamide-treated eye, the mean 24-hour IOP was $14.6 \pm 2.2 \mathrm{~mm} \mathrm{Hg}$ before brinzolamide administration and was significantly reduced by $10.3 \%$ to $13.1 \pm 1.9 \mathrm{~mm} \mathrm{Hg}$ after brinzolamide administration $(\mathrm{p}=0.001)$. In brinzolamideuntreated eye, the mean IOP was $13.5 \pm 2.8 \mathrm{~mm} \mathrm{Hg}$ and $13.8 \pm 2.7 \mathrm{~mm} \mathrm{Hg}$, respectively, showing no significant changes.

\section{Maximum and Minimum IOPs and a Range of Fluctua- tions in IOP}

In brinzolamide-treated eye, the maximum IOP was $17.2 \pm 2.9 \mathrm{~mm} \mathrm{Hg}$ before brinzolamide administration and was significantly reduced to $15.6 \pm 2.2 \mathrm{~mm} \mathrm{Hg}$ after brinzolamide administration $(\mathrm{P}=0.02)$. The minimum IOP was $11.6 \pm 2.0 \mathrm{~mm} \mathrm{Hg}$ before brinzolamide administration and was significantly reduced to $10.3 \pm 1.9 \mathrm{~mm} \mathrm{Hg}$ after brinzolamide administration $(\mathrm{P}=0.04)$. The range of fluctuations in IOP was $5.4 \pm 1.6 \mathrm{~mm} \mathrm{Hg}$ before brinzolamide administration and $5.4 \pm 1.4 \mathrm{~mm} \mathrm{Hg}$ after brinzolamide administration, showing no significant differences.
In brinzolamide-untreated eye, the maximum IOP was $15.7 \pm 3.3 \mathrm{~mm} \mathrm{Hg}$ before brinzolamide administration and $16.5 \pm 2.8 \mathrm{~mm} \mathrm{Hg}$ after brinzolamide administration, showing no significant differences. Both the minimum IOP and the range of fluctuations in IOP also showed no significant differences before and after brinzolamide administration: the minimum IOP was $11.3 \pm 2.4 \mathrm{~mm} \mathrm{Hg}$ versus $11.4 \pm 2.3 \mathrm{~mm} \mathrm{Hg}$ and the range of fluctuations in IOP was $4.7 \pm 1.7 \mathrm{~mm} \mathrm{Hg}$ versus $5.2 \pm 1.0 \mathrm{~mm} \mathrm{Hg}$.

\section{Diurnal Fluctuations in IOP After Brinzolamide Admini- stration}

The additional administration of brinzolamide resulted in a greater reduction in IOP at all the measurement points compared to latanoprost alone. Significant reductions were observed at $0 \mathrm{PM}, 4 \mathrm{PM}, 0 \mathrm{AM}, 3 \mathrm{AM}, 6 \mathrm{AM}$, and $8 \mathrm{AM}$ (Fig. 1a). On the other hand, there were no significant changes in IOP in brinzolamide-untreated eye at any measurement points (Fig. 1b). The percent reductions in IOPs at 6 $\mathrm{PM}$ and $6 \mathrm{AM}$, time points immediately before brinzolamide administration, were lower than those at measured times just after brinzolamide administration (Fig. 2). Comparison of IOP values over 24 hours between brinzolamide-treated and brinzolamide-untreated eyes showed that IOP in brinzolamide-treated eye was lower at all the measurement points and there were significant differences in IOP at all the measurement points except for at $0 \mathrm{PM}$ and $10 \mathrm{PM}$ (Fig. 3). Comparison of the percent reduction in IOPs between daytime ( 8 AM, 10 AM, 0 PM, 2 PM, 4 PM) and nighttime (8 PM, 10 PM, 0 AM, 3 AM) showed no significant differences, with a mean of $9.5 \pm 4.7 \%$ and $10.3 \pm 5.6 \%$, respectively.

\section{DISCUSSION}

IOP-lowering therapy is the most effective approach for treatment of glaucoma, regardless of pattern of glaucoma. Though surgical treatment can drastically lower IOP, it may sometimes cause a serious complication. Laser therapy has an excellent safety profile but poor efficacy. For these reasons, ophthalmic solutions are often used as a first choice treatment for glaucoma. While there are a growing number of therapeutic options for glaucoma, latanoprost is the most widely used drug nowadays. Latanoprost has a beneficial effect of lowering IOP over 24 hours and it causes fewer adverse reactions [4]. However, there are many patients who can not be fully treated with latanoprost alone.

It has been shown that while $\beta$-blockers cause fewer local adverse reactions and are effective in lowering IOP during daytime, this IOP-lowering effect is impaired during nighttime [10-12]. Therefore, there has been a need for drugs that can lower IOP even at night in order to maintain lower IOP over 24 hours. Brinzolamide is a carbonic anhydrase inhibitor that suppresses formation of aqueous humor [6, 13]. There have been some reports that it causes fewer local adverse reactions and lowers IOP over 24 hours [6, 9]. As the IOP-lowering effect of latanoprost is mediated by the uveoscleral pathway, we expected that combination therapy with latanoprost and brinzolamide might produce IOP-lowering effect over 24 hours, including during nighttime. The results of the present study showed that brinzolamide further enhanced the IOP-lowering effect of latanoprost over 24 hours, suggesting a potential for IOP-lowering therapy with more 


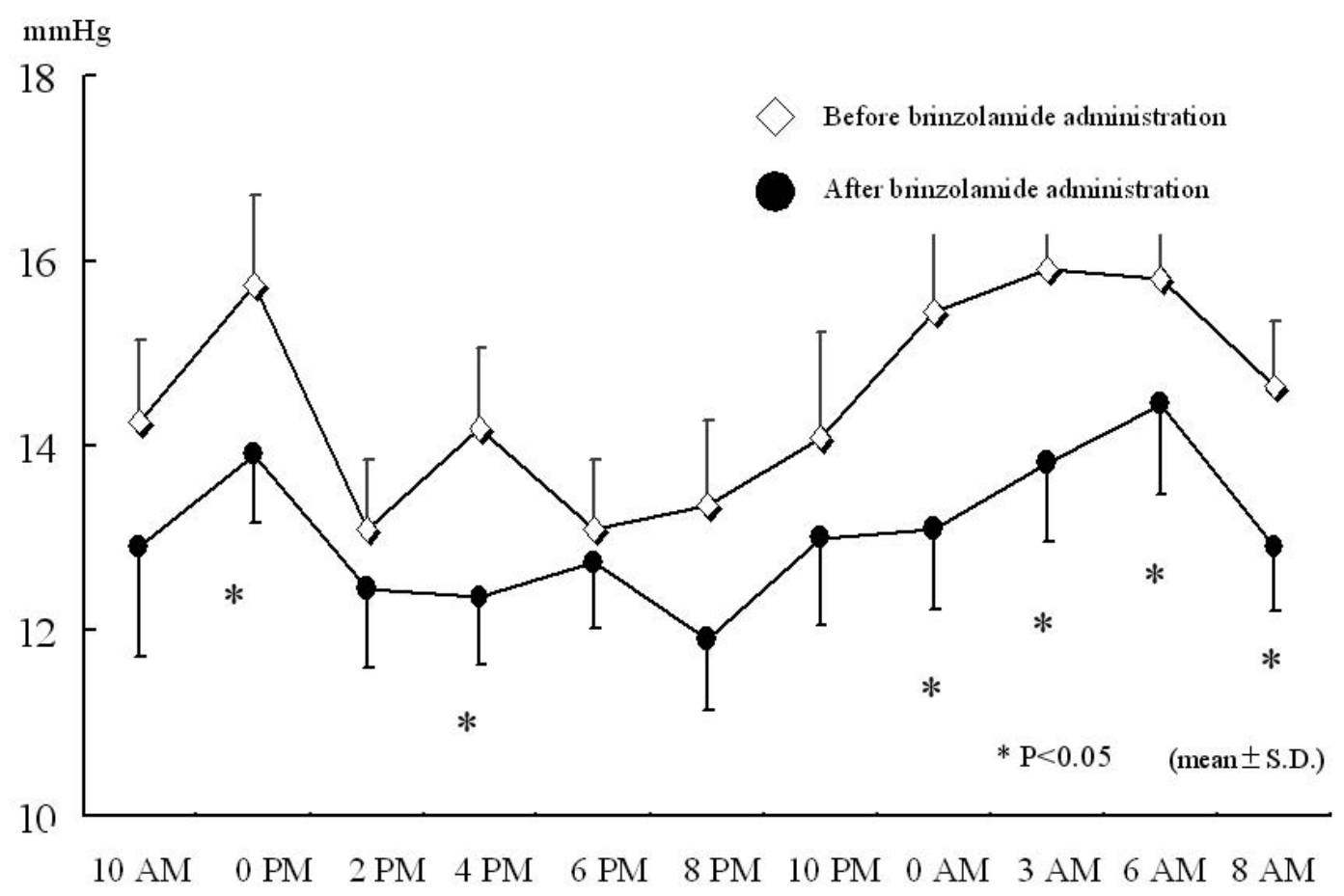

(b)

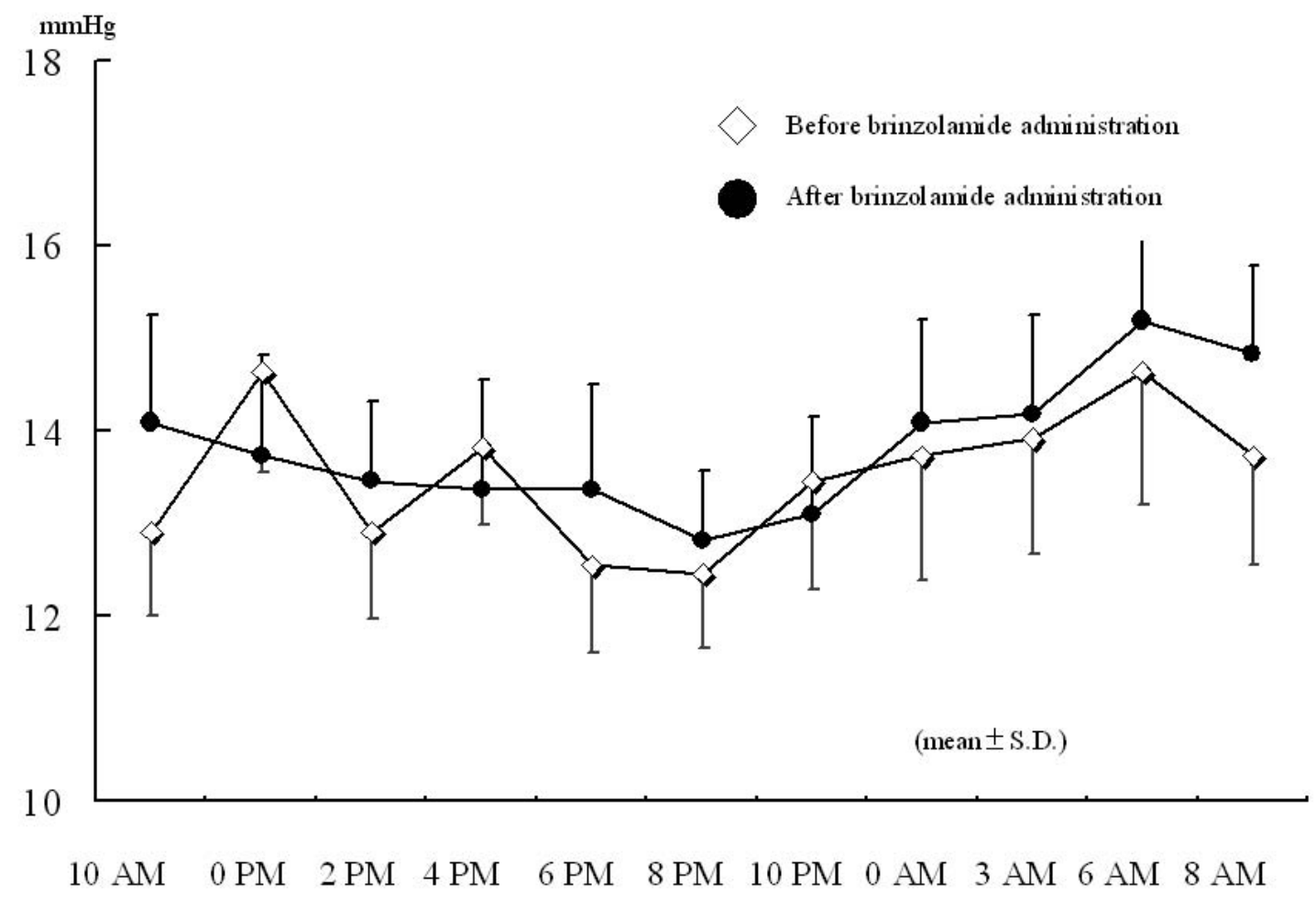

Fig. (1). Changes in IOP over 24 hours after brinzolamide administration. (a) Brinzolamide-treated eye. (b) Brinzolamide-untreated eye. $* \mathrm{P}<0.05$, all data expressed as mean $\pm \mathrm{SD}$. Open diamond: before brinzolamide administration, closed circle: after brinzolamide administration. 


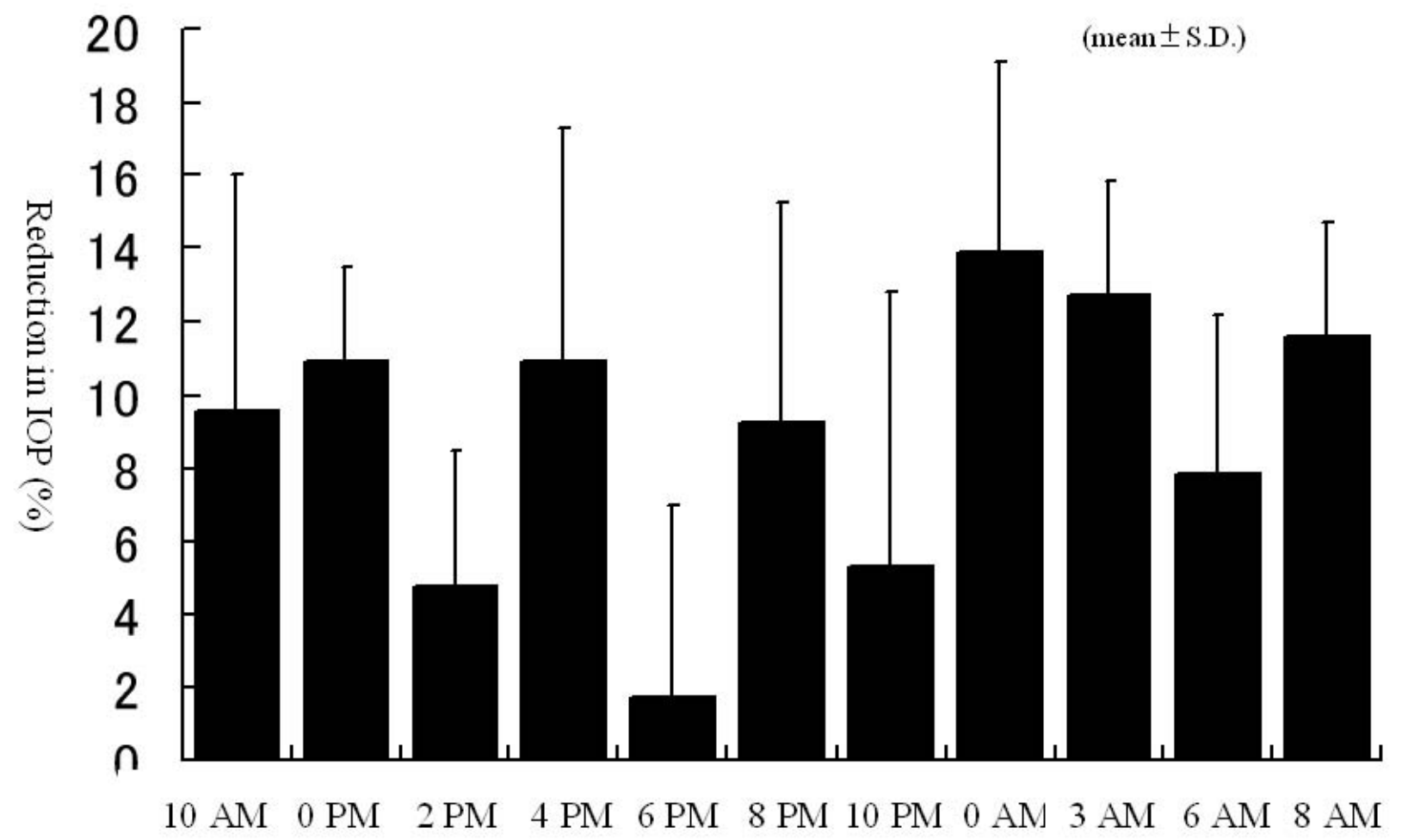

Fig. (2). Percent reduction in IOPs over 24 hours after brinzolamide administration. All data expressed as mean \pm SD.

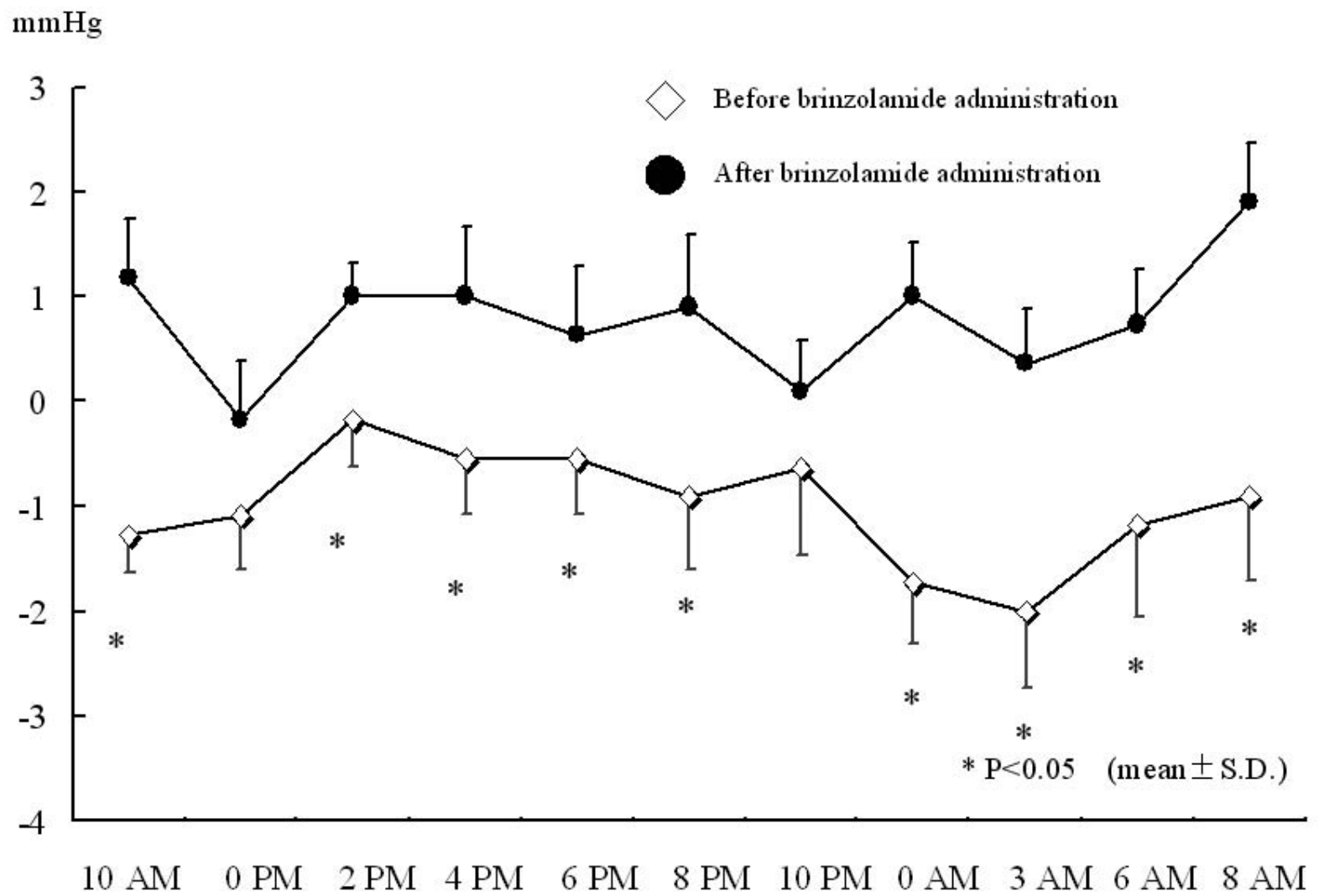

Fig. (3). Difference in IOP between right and left eyes before and after brinzolamide administration. ${ }^{*} \mathrm{P}<0.05$, all data expressed as mean \pm SD. 
consistency. In Japan, there are many patients with NTG [5] and there is a need for controlling IOP in patients in less than fourteen $\mathrm{mmHg}$. Among 11 patients in our study, while only 2 patients achieved consistent IOP control of $13 \mathrm{~mm} \mathrm{Hg}$ or lower with latanoprost, 6 patients $(54.5 \%)$ achieved IOP control of $13 \mathrm{~mm} \mathrm{Hg}$ or lower after the additional administration of brinzolamide. This suggests that these two drugs can maintain IOP at $13 \mathrm{~mm} \mathrm{Hg}$ or lower in more than a half of the patients and that the combination therapy with latanoprost and brinzolamide may be useful in treating NTG.

This additive effect of the two drugs may be explained by their difference in mechanism of lowering IOP: latanoprost by promoting uveoscleral aqueous outflow and brinzolamide by suppressing formation of aqueous humor.

The percent reduction in IOPs tended to decrease immediately before brinzolamide administration, suggesting a need for adjusting time of administration of other ophthalmic solutions in order to achieve IOP-lowering effect over 24 hours. No patients in the present study discontinued brinzolamide administration due to drug-related adverse reactions. This suggests that brinzolamide may be an excellent ophthalmic solution that causes fewer adverse reactions. The additional administration of brinzolamide resulted in an average reduction in IOP of $10.3 \%$, demonstrating an equivalent effect to oral carbonic anhydrase inhibitors [7]. While long-term use of oral carbonic anhydrase inhibitors should be avoided due to severe systemic adverse reactions [14], brinzolamide is associated with a lower incidence of adverse reactions and maintains the IOP-lowering effect over 24 hours, suggesting that brinzolamide may be effective in treating glaucoma.

This study has a limitation mentioned below. Since the number of participates in this study is relatively small, it is required to perform further study to confirm our results with much more sample size. It is much better if we can compare the difference in the diurnal change of IOP between brinzolamide alone and a combination of latanoprost and brinzolamide. However, brinzolamide is approved to be used as a second line drug by the government.

\section{REFERENCES}

[1] Whitson JT. Glaucoma: a review of adjunctive therapy and new management strategies. Expert Opin Pharmacother 2007; 8: 323749.

[2] Liu JH, Kripke DF, Twa MD, et al. Twenty-four-hour pattern of intraocular pressure in young adults with moderate to severe myopia. Invest Ophthalmol Vis Sci 2002; 43: 2351-5.

[3] Liu JH, Zhang X, Kripke DF. Weinreb RN. Twenty-four-hour intraocular pressure pattern associated with early glaucomatous changes. Invest Ophthalmol Vis Sci 2003; 44: 1586-90.

[4] Orzalesi N, Rossetti L, Invernizzi T, Bottoli A, Autelitano A. Effect of timolol, latanoprost, and dorzolamide on circadian IOP in glaucoma or ocular hypertension. Invest Ophthalmol Vis Sci 2000; 41: 2566-73.

[5] Iwase A, Suzuki Y, Araie M, et al. The prevalence of primary open-angle glaucoma in Japanese: The Tajimi Study. Ophthalmology $2004 ; 111: 1641-8$.

[6] Silver LH. Clinical efficacy and safety of brinzolamide (Azopt), a new topical carbonic anhydrase inhibitor for primary open-angle glaucoma and ocular hypertension. Brinzolamide Primary Therapy Study Group. Am J Ophthalmol 1998; 126: 400-8.

[7] Sugrue MF. Pharmacological and ocular hypotensive properties of topical carbonic anhydrase inhibitors. Prog Retin Eye Res 2000; 19: 87-112.

[8] Cvetkovic RS, Perry CM. Brinzolamide : a review of its use in the management of primary open-angle glaucoma and ocular hypertension. Drugs Aging 2003; 20: 919-47.

[9] Nakamoto K, Yasuda N. Effect of concomitant use of latanoprost and brinzolamide on 24-hour variation of IOP in normal-tension glaucoma. J Glaucoma 2007; 16: 352-7.

[10] Larsson LI, Konstas AG, Mylopoulos N, et al. Intraocular pressure over 24 hours after repeated administration of latanoprost $0.005 \%$ or timolol gel-forming solution $0.5 \%$ in patients with ocular hypertension. Ophthalmology 2001; 108: 1439-44.

[11] Costagliola C, Parmeggiani F, Virgili G, et al. Circadian changes of intraocular pressure and ocular perfusion pressure after timolol or latanoprost in Caucasians with normal-tension glaucoma. Graefes Arch Clin Exp Ophthalmol 2007; 15: 15.

[12] Konstas AG, Mylopoulos N, Karabatsas CH, et al. Diurnal intraocular pressure reduction with latanoprost $0.005 \%$ compared to timolol maleate $0.5 \%$ as monotherapy in subjects with exfoliation glaucoma. Eye 2004; 18: 893-9.

[13] Ingram CJ, Brubaker RF. Effect of brinzolamide and dorzolamide on aqueous humor flow in human eyes. Am J Ophthalmol 1999; 128: 292-6.

[14] Hoyng PF, van Beek LM. Pharmacological therapy for glaucoma: a review. Drugs 2000; 59: 411-34. 